\title{
Effect of Self-field and Loosening of Stacked Superconducting Tapes on Critical Current of a Single Pancake Coil
}

\author{
Ashish Agrawal, Abhinav Kumar
}

\begin{abstract}
Research on superconducting systems is getting pace due to the increasing demand of efficient machines to fulfill the need of the society. Applications like motors, transformers and magnetic energy storage systems involve the pancake coils tightly bounded with the superconducting tapes. Moreover, all such devices are sensitive to the number of turns and operating current. Critical current of the superconducting tape is most important parameter while designing such machines as if operating current exceeds this value it will turn to a normal conductor. Critical current of the tape is further depending upon the operating temperature, external and self-field. In this work, effect of both self-field and degree of tightness or looseness on the critical current of the tape has been studied. The results showed that the critical current of the tape is significantly affected by both self-field and the inter-distance among the adjacent tapes.
\end{abstract}

Keywords: Superconducting tape, critical current, pancake coils, Superconductivity, High temperature superconductors.

\section{INTRODUCTION}

$\mathrm{H}$ igh temperature superconducting tapes are widely employed in various applications including high voltage power transmission, generators, motors, energy storage, and transformers etc. These superconducting systems can be implemented in various engineering fields like electric power transmission, power grid stabilization, ship propulsion, Maglev trains propulsion, electric aircrafts, solar and wind energy storage systems. Coated conductors can be used as long cables, single pancake coils and double pancake coils having circular or race-track configuration [1]-[10]. Coated conductors are used due to their capability to handle large currents (helpful in power transmission cables) and magnetic fields (energy storage systems like SMES). A tape can be used as single tape or in a stack of tapes forming Roebel configuration which is widely employed in various studies [6][10]-[14]. Pancake coils are used in various applications including motor winding [15]-[17], magnets (solenoidal or toroidal) [3][18]-[20] where these are piled up on top of the other to generate the required field. In order to retain the superconducting nature of the tape, it has to be cooled below critical temperature which limits the amount of current (termed as critical current of the tape) that can carry through the tape. It has been found that this current is significantly

Revised Manuscript Received on December 02, 2019

* Correspondence Author

Ashish Agrawal, Department of Mechanical Engineering, Madanapalle Institute of Technology and Science, Madanapalle, India. Email: drashishagrawal@mits.ac.in

Abhinav Kumar*, School of Mechanical Engineering, Lovely Professional University, Phagwara, India. Email: drabhinav@ieee.org affected due to the presence of either external magnetic fields or self-fields [6][14][21]-[23]. Thus, it becomes essential to evaluate the critical current $\left(I_{c}\right)$ dependency on the magnetic field as estimation of $I_{c}$ can directly affect the design and optimization of the superconducting systems. Though, almost all superconducting tape manufacturers have defined the $I_{c}$ characteristics with $0 \mathrm{~T}$ self-filed. Also, there is lack of information on the effect of external field to the $I_{c}$ of the tape. Moreover, the effect of neighboring tapes or coils has not been considered in the general information catalogue as the $I_{c}$ can vary depending on the temperature and type of engineering applications. Researchers have done studies where they have considered the effect of self-field for estimating the critical current of the superconducting tape [14][21][22]. Most of these studies consider cables with low aspect ratios or having small circular cross-sectional area. Few studies have been performed where cable consisting superconducting tapes with high aspect ratios where critical current of Roebel cables (used in transformer windings) have been estimated [14][22]. These models have provided fair results however, they need comparative and iterative processes to evaluate the $I_{c}$ and there is a need to consider uniform current distribution within each superconductor. A more accurate way to estimate critical current of the tape wounded as a coil or cable is to incorporate the critical current dependency of the superconducting tape or coil on the local flux density $J_{c}(\boldsymbol{B})$. Kim model [24] is usually used to describe the critical current density dependency on the local magnetic flux density $J_{c}(\boldsymbol{B})$. This model is also employed to estimate the $\mathrm{AC}$ losses among superconducting devices using various formulations [25]-[28]. In order to model the resistivity of the superconducting material, generally $E-J$ power law is used. The numerical model has been solved for AC losses where Kim model is integrated with $E-J$ power law and the importance of local flux density has been considered.

In this work, the same DC model as developed by Grilli and Zermeno et al. [22] using Comsol has been incorporated in order to estimate the critical current with greater accuracy of the superconducting systems such as electric aircraft propulsion motors, superconducting magnetic energy storage devices, power transmission cables etc. The model has been developed where single pancake coil having 108 turns and the critical current of the tape is $330 \mathrm{~A}$ at $77 \mathrm{~K}$ [29].

The developed model is having an additional variable $P$ which is designed to be uniform in each domain to ensure the non-linear $E$-J power law relationship. 
This model works very well and it is easy to implement in order to estimate the critical current of the multi-turned pancake coils. The of tight and loose winding has been examined and the results showed that there is a significant variation in the $I_{c}$ of the tape due to self-field and the gap between adjacent layers or turns of pancake coil.

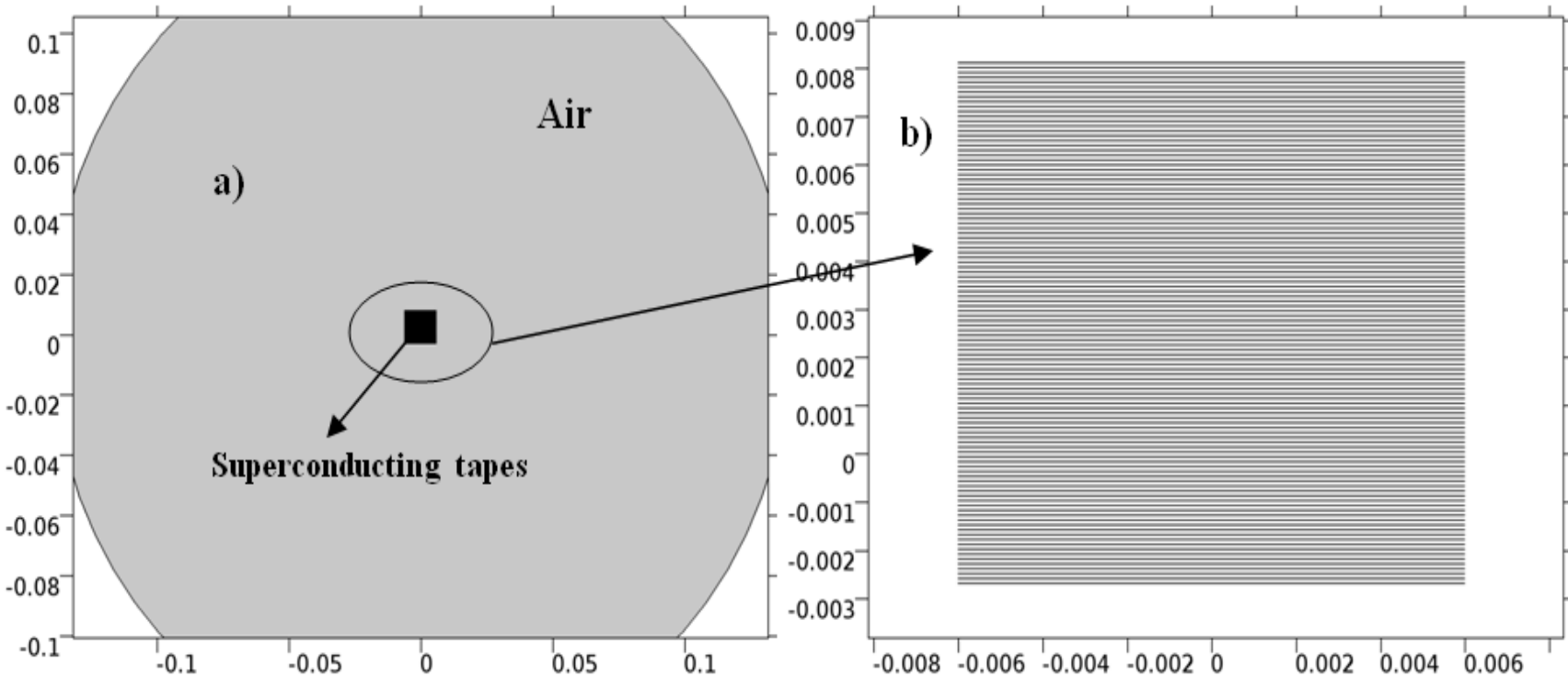

Fig. 1 Geometrical representation of the 2D model

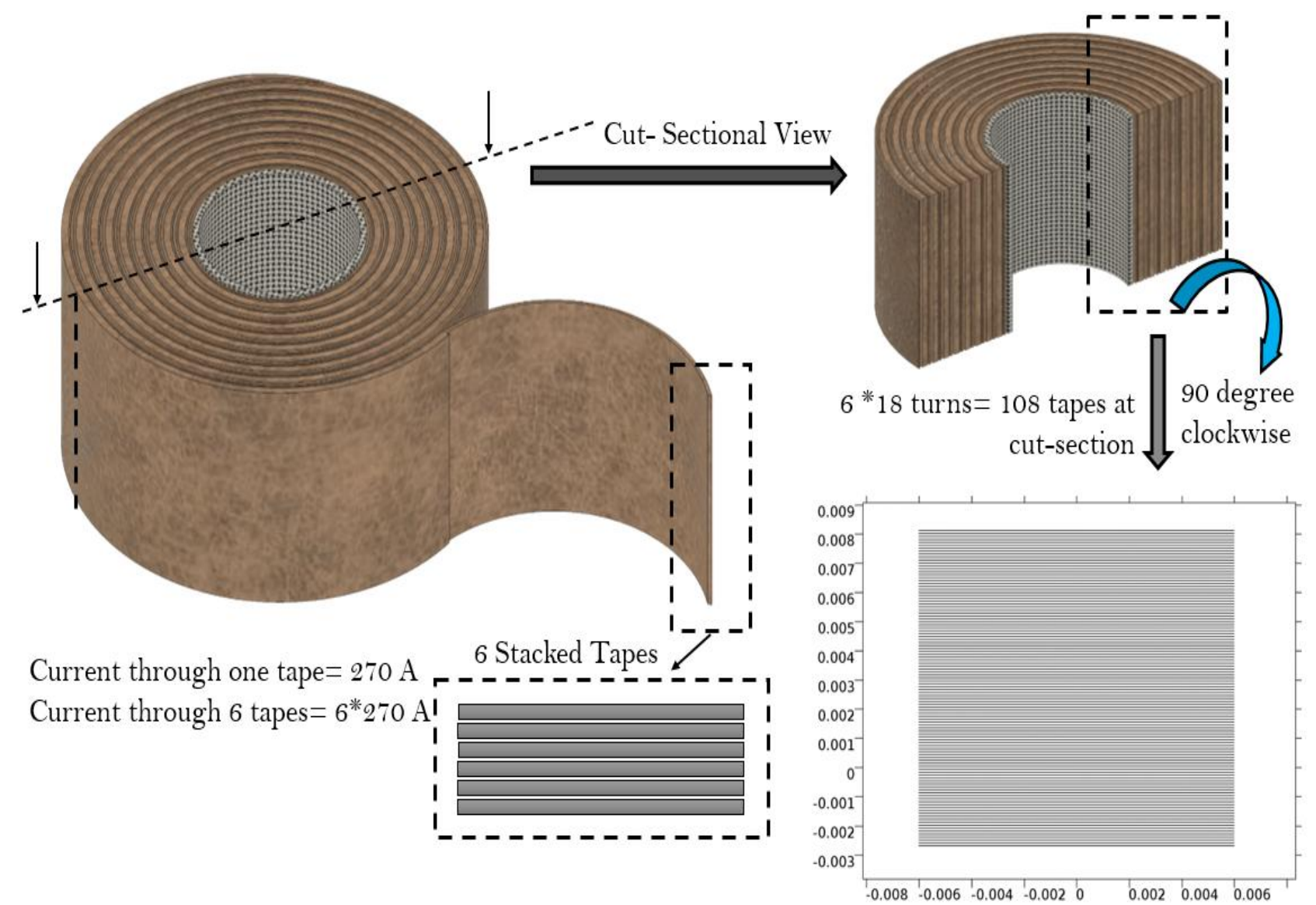

Fig. 2 Arrangement of 108 tapes (6 stacked tapes with 18 turns)

A 2D model comprising air and superconducting tapes is shown in Fig. 1 where Fig. 1 (b) shows the arrangement of 108 turns around a pancake. Only superconducting layer has been considered among the tape as most of the current is assumed to be flow through it. 
Fig. 2 shows the detailed description of the numerical model approximation. The width and thickness of the superconducting layer is $12 \mathrm{~mm}$ and $1 \mathrm{e}-6 \mu \mathrm{m}$ respectively. Table I show the modelling parameters used in the present study. Computational problem is solved using Ampere's law whose differential form is given by:

$$
\begin{aligned}
& \nabla \times H=J \\
& B=\nabla \times A \\
& J=\sigma E \\
& \rho_{H T S}=\frac{E_{c}}{J_{c}}\left|\frac{J}{J_{c}}\right|^{n-1}
\end{aligned}
$$

To model the resistivity of superconducting layer E-J power law has been used with index, $n=30$. Comsol Multiphysics software package is used for the non-time dependent analysis. The developed model is having an additional variable $P$ which is designed to be uniform in each domain to ensure the non-linear $E-J$ power law relationship. This model works very well and it is easy to implement in order to estimate the critical current of the multi-turned pancake coils.

\section{RESULTS AND DISCUSSIONS}

The critical current for the assumed tape is $330 \mathrm{~A}$ and as 108 tapes are stacked around the coil thus the critical current for all tapes should equal to 35,640 A without considering self-field effect. However, when the effect of self-field is considered its value found to equal to $29,671 \approx 275 \mathrm{~A}$ for a single tape which was initially 330 A. This shows that the self-field has significant effect on the critical current. Also, the effect of the gap among the adjacent turns has been varied in order to evaluate the effect of the loosening or tightening of the SC tapes. Fig. 3 shows that with the increase in the inter-distance among tapes, the critical current is found to increase. However, the magnetic flux density is found to decrease with the inter-distance among tapes and which results in less dense fields.

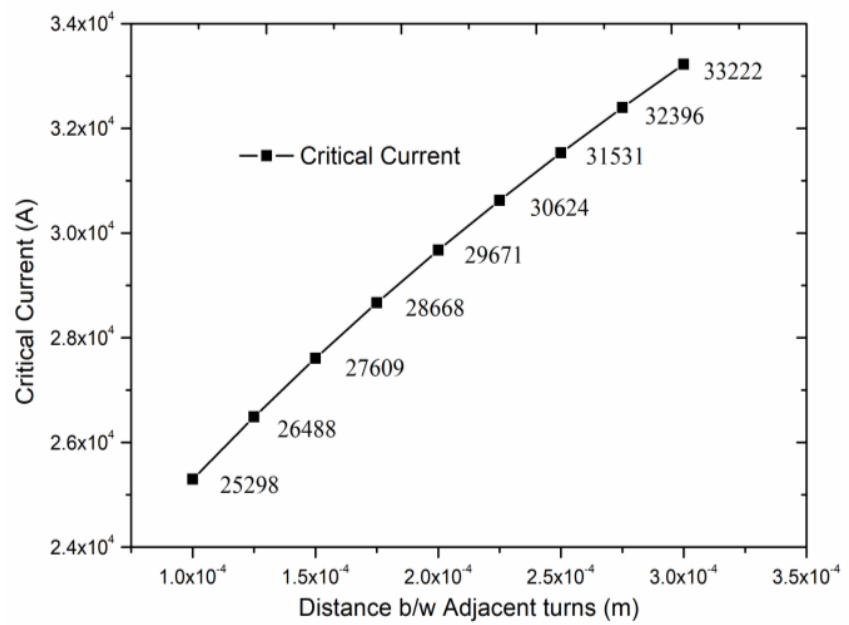

Figure 3 Critical Current vs inter-distance among tapes

Therefore, the inter-distance among the tapes is a crucial factor while tightening the coils as loosening can affect the magnetic flux density significantly. Such situations usually impact the performance of magnetic energy storage systems where higher denser fields are generally required to have higher power densities. Also, the effect of number of tapes may also affect the critical current and magnetic flux densities due to self-field however in this work that aspect has not been taken into account.

Table- I: Model Parameters

\begin{tabular}{|l|l|}
\hline \multicolumn{1}{|c|}{ Parameters } & \multicolumn{1}{c|}{ Data } \\
\hline Number of turns & 108 \\
\hline Width of the tape & $12 \mathrm{~mm}$ \\
\hline Thickness of the tape & $1 \mu \mathrm{m}$ \\
\hline Inter-distance among tape & 1 to $3 \times 10^{-4} \mathrm{~m}$ \\
\hline Index, $\mathrm{n}$ & 30 \\
\hline Critical Electric field & $10^{-4} \mathrm{~V} / \mathrm{m}$ \\
\hline Constant, $\mathrm{k}$ & 0.29515 \\
\hline Magnetic field, B0 & $42.65 \mathrm{mT}$ \\
\hline
\end{tabular}

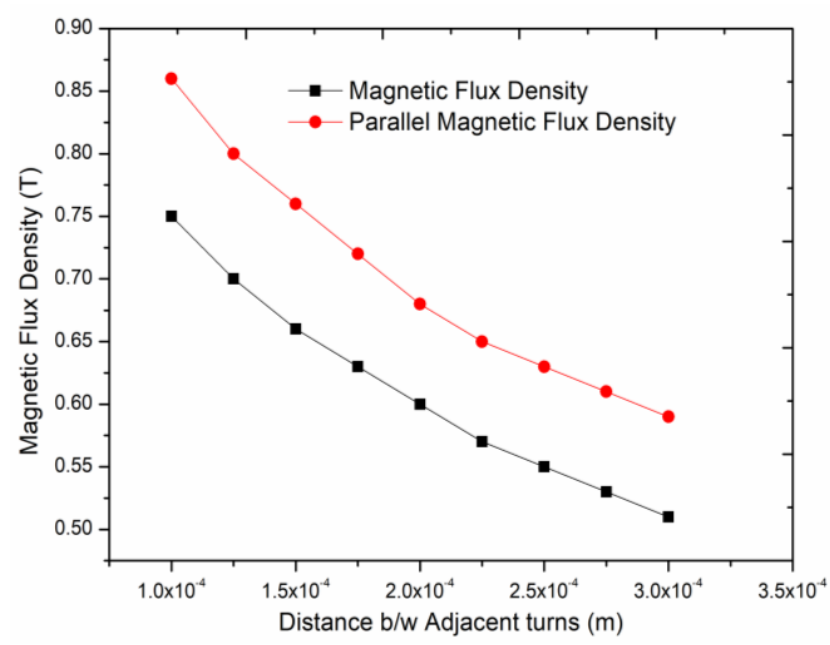

Figure 4 Magnetic flux density vs inter-distance among tapes

\section{CONCLUSION}

The developed 2D model is a fair approximation for this problem where the effect of self-field and degree of looseness of windings on critical current of the superconducting tape has been studied. The model predicts that there is a significant effect of both self-field and degree of looseness of windings on the critical current thus these measures has to be taken into account before designing the pancake coils for superconducting applications.

\section{ACKNOWLEDGMENT}

Authors would like to thank Dr. Victor Manuel Rodriguez Zermeno for his technical support in understanding the concept.

\section{REFERENCES}

1. M. Park et al., "Conceptual Design of HTS Magnet for a 5 MJ Class SMES," vol. 18, no. 2, pp. 750-753, 2008.

2. S. Kwak et al., "Design of HTS Magnets for a 2 . 5 MJ SMES," vol. 19, no. 3, pp. 1985-1988, 2009.

3. A. Kumar and R. Kaur, "Electromagnetic analysis of $1 \mathrm{MJ}$ class of high temperature superconducting magnetic energy storage (SMES) coil to be used in power applications," vol. 050003, p. 050003, 2018.

4. A. Morandi et al., "Design and Comparison of a 1-MW / 5-s HTS SMES With Toroidal and Solenoidal Geometry," vol. 26, no. 4, pp. $1-6,2016$. 
5. T. Takematsu et al., "Degradation of the performance of a YBCO-coated conductor double pancake coil due to epoxy impregnation," Phys. C Supercond. its Appl., vol. 470, no. 17-18, pp. 674-677, 2010.

6. F. Grilli, M. Vojen`, A. Kario, and V. Zerme, "Estimation of Self-Field Critical Current and Transport-Magnetization AC Losses of Roebel Cables," pp. 1-5, 2015.

7. Q. Wang et al., "High Temperature Superconducting YBCO Insert for 25 T Full Superconducting Magnet," vol. 25, no. 3, pp. 3-7, 2015.

8. V. M. R. Zermeno, F. Grilli, and F. Sirois, "A full 3D time-dependent electromagnetic model for Roebel cables," Supercond. Sci. Technol., vol. 26 , no. 5 , p. 52001, 2013.

9. Z. Jiang et al., "Magnetization Loss in REBCO Roebel Cables," vol. 28, no. 3,2018 .

10. E. Pardo and F. Grilli, "Numerical simulations of the angular dependence of magnetization AC losses : coated conductors , Roebel cables and," vol. 014008, 2012.

11. W. Goldacker, F. Grilli, E. Pardo, and A. Kario, "Roebel cables from REBCO coated conductors: a one-century-old concept for the superconductivity of the future," vol. 093001.

12. V. M. R. Zermeno, F. Grilli, and F. Sirois, "A full 3D time-dependent electromagnetic model for Roebel cables," Supercond. Sci. Technol., vol. 26, no. 5, 2013.

13. F. Grilli, M. Vojen`, A. Kario, and V. Zerme, "HTS Roebel Cables: Self-Field Critical Current and AC Losses under Simultaneous Application of Transport Current and Magnetic Field," vol. 8223, no. c, pp. 1-5, 2016

14. F. Grilli, V. M. R. Zerme, E. Pardo, M. Vojen`, and A. Kario, "Self-field Effects and AC Losses in Pancake Coils Assembled from Coated Conductor Roebel Cables," pp. 1-5.

15. M. Furuse, M. Yoshikawa, Y. Itoh, S. Fukui, and T. Nakamura, "Fabrication and Testing of Racetrack-Shaped Double-Pancake Coil for Stator Windings of Induction-Synchronous Motor," vol. 25, no. 3, 2015.

16. W. Y. Li, X. J. Niu, H. Su, W. Chen, S. S. Peng, and J. Zheng, "A Study on HTS Double Pancake Coil for Electric Machine," pp. 177-179, 2013

17. K. Nagao, T. Nakamura, H. Sugimoto, and T. Morishita, "Synchronous motor with HTS-2G wires."

18. K. C. Seong et al., "Development of a 600 kJ HTS SMES," vol. 468, pp 2091-2095, 2008.

19. A. Morandi, M. Fabbri, B. Gholizad, F. Grilli, F. Sirois, and V. M. R. Zermeño, "Design and Comparison of a 1-MW/5-s HTS SMES With Toroidal and Solenoidal Geometry," IEEE Trans. Appl. Supercond., vol. 26 , no. 4, pp. 1-6, 2016

20. A. Kumar, J. V. M. L. Jeyan, and A. Agarwal, "Numerical analysis on $10 \mathrm{MJ}$ solenoidal high temperature superconducting magnetic energy storage system to evaluate magnetic flux and Lorentz force distribution," Phys. C Supercond. its Appl., vol. 558, pp. 17-24, 2019.

21. F. Grilli, F. Sirois, S. Member, V. M. R. Zermeño, and M. Vojen`, "Self-Consistent Modeling of the I c of HTS Devices : How Accurate do Models Really Need to Be?," vol. 24, no. 6, 2014.

22. V. Zermeño, F. Sirois, M. Takayasu, M. Vojenciak, A. Kario, and F. Grilli, "A self-consistent model for estimating the critical current of superconducting devices," Supercond. Sci. Technol., vol. 28, no. 8, p. 85004.

23. J. Fleiter, A. Ballarino, L. Bottura, and P. Tixador, "Electrical characterization of REBCO Roebel cables," Supercond. Sci. Technol., vol. 26 , no. 6 , p. $65014,2013$.

24. K. P. Thakur, A. Raj, E. H. Brandt, J. Kvitkovic, and S. V Pamidi, "Frequency-dependent critical current and transport ac loss of superconductor strip and Roebel cable," vol. 065024, 2011.

25. W. Yuan, A. M. Campbell, and T. A. Coombs, "A model for calculating the AC losses of second-generation high temperature superconductor pancake coils," Supercond. Sci. Technol., vol. 22, no. 7, p. 75028, 2009.

26. E. Pardo, J. Šouc, and J. Kováč, "AC loss in ReBCO pancake coils and stacks of them: modelling and measurement," Supercond. Sci. Technol., vol. 25, no. 3, p. 35003, 2012.

27. W. Yuan, A. M. Campbell, and T. A. Coombs, "Ac losses and field and current density distribution during a full cycle of a stack of superconducting tapes," J. Appl. Phys., vol. 107, no. 9, p. 93909, 2010.

28. A. Kumar, A. Agrawal, and J. M. L. Jeyan, "A Numerical Model Comprising the Effect of Number of Turns on AC Losses in 2G HTS Coated Conductor at 77K using H-formulations," in 2019 IEEE 2nd International Conference on Power and Energy Applications (ICPEA), 2019 , pp. $115-118$

29. S. H. T. Superconductor, "SuperPower ${ }^{\circledR} 2$ G HTS Wire Specifications," pp. 2-5, 2000

\section{AUTHORS PROFILE}

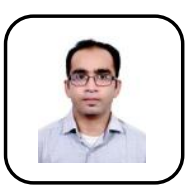

Dr. Ashish Agrawal is an Assistant Professor at the Department of Mechanical Engineering in Madanapalle Institute of Technology and Science in Madanapalle, Andhra Pradesh in India. He obtained his Masters and $\mathrm{PhD}$ from Indian Institute of Technology, Kanpur in 2010 and 2017 respectively. He graduated in Mechanical Engineering from Madhav Institute of Technology and Science in Gwalior, Madhya Pradesh in India. Prior joining to MITS Madanapalle, he has worked as an Assistant Professor at Lovely Professional University in Punjab. Broadly his research interests lie in computational and experimental heat transfer and fluid dynamics. More specifically, his research sears include heat transfer in rotary kilns, electronics cooling, superconducting magnetic energy storage system and vortex tube cooling system.

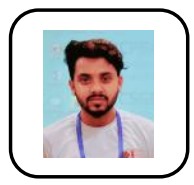

Abhinav Kumar is pursuing his Doctorate in Mechanical Engineering from Lovely Professiona University, Punjab, India. He has completed his master's in thermal engineering specialization from Lovely Professional University in 2014 and bachelor's in Mechanical Engineering from Punjab Technical University in 2011. He has published many SCI and Scopus indexed articles in various journals and has attended many international conferences. His area of research is vast including superconducting magnetic energy storage systems, superconducting fault current limiters, high temperature superconducting cable design \& cooling, cryogenic fluid properties, nozzle cooling, computational fluid dynamics and solar energy storage systems. He is one of the reviewers for SCI and Scopus indexed journals and conferences. $\mathrm{He}$ has been awarded with University Academic Honor and University Honor Roll for his excellent academic records. He is holding Student IEEE and life membership of Indian Society of Heat and Mass Transfer (ISHMT). 